\title{
Exploring the expansive dimensions of education
}

\author{
Janet R. Reid ${ }^{1}$ (i) Peter J. Strouse ${ }^{2}$ \\ Received: 10 April 2020 / Revised: 10 April 2020 / Accepted: 28 April 2020 / Published online: 14 May 2020 \\ (C) Springer-Verlag GmbH Germany, part of Springer Nature 2020
}

\section{Introduction}

What comes to mind when you hear the word education? Lectures? PowerPoint slides? Metrics? Competencies? Perhaps, for some, it is all these things, but for others it may be something different. In a specialty where much of the learning takes place at the point-of-care, it is surprising that radiology has been slow to reach across the aisle to glean from education experts whose definition of education is much more expansive and exciting. As pediatric radiologists, we are some of the most dedicated teachers in radiology. It is time for us to partner with education leaders to create a new flagship for radiology education that dedicates space in our lead journal for focus pieces on an expansive definition of education. We will address, among other topics: mentors who know how to engender engagement, teaching to mastery, how and when to use technology to have a positive impact on teaching, and case studies in impactful education research. The following is a preview of upcoming content in the new education focus of Pediatric Radiology.

\section{Mentoring}

Many of us can recall a favorite mentor during training. If we think of why that person had such a positive impact, we will note that he or she gave us individual attention and displayed unconditional regard. As experts in radiology education, it is important for us to consider each teacher-student dyad a unique combination. By borrowing from child development

Janet R. Reid

reidj@email.chop.edu

1 Department of Radiology, Children's Hospital of Philadelphia, University of Pennsylvania, 324 South 34th St., 3NW 13, Philadelphia, PA 19104, USA

2 Section of Pediatric Radiology, C. S. Mott Children's Hospital, Department of Radiology, Michigan Medicine, Ann Arbor, MI, USA theory, we can learn to be incredibly effective in our teaching of adult learners in a psychologically safe environment. With this education expertise, we will find that we have a profound impact on many more students, and that our modeling has the power to produce the next generation of education experts and leaders.

\section{Storytelling}

If a picture is worth a thousand words, then what is the value of a story? In fact, storytelling is an extremely effective tool that is as old as human civilization. Its benefits lie beyond the message, and when initiated by the teacher, it introduces a level of vulnerability that can be incredibly powerful in jumpstarting engagement. Stories told well are both captivating and versatile in being able to transmit facts, messages, concepts and emotions. A well-rehearsed story leads to greater learning retention than a 45-min PowerPoint presentation.

\section{Mastery}

Our practice of medicine has become very metrics-driven, and so has our practice of education. An important question to ask as teachers of pediatric radiology is this: Are we are content with teaching to competencies or should we be looking for something greater? Is it possible for us to rethink radiology education to aim for mastery so that our graduates start their careers having greater impact as true consultants? What would it take to stretch toward mastery in pediatric radiology education?

\section{Technology}

Technology has become the buzzword in education and has come to be expected in certain learning environments. It includes audience response systems, polling software, 
enhancements to PowerPoint, electronic games, etc. But those across the aisle would teach us that the content and message are still more important than the medium, and that the medium can actually destroy the message if chosen poorly. Imagine using an audience response system in a 1:1 conversation with a student or bringing PowerPoint slides to a church picnic! Technology used judiciously should add power to a teaching session, augmenting engagement. It serves as a picture frame - not the picture.

\section{Research}

Perhaps one of the most exciting opportunities in radiology education will be found in research opportunities and research leadership, studying education itself. Again, borrowing from our education colleagues, as well as those working in quality improvement, pediatric radiology has ample opportunity to conduct research that proves learning value as well as learning retention. There is an opportunity to then tie results to changes in management and patient outcomes, with potential to transform the way we teach and learn. To accomplish this, we must become experts in the qualitative and mixed methodologies that are most commonly employed in education research. In addition, we must cultivate reviewers with this same expertise.

\section{Focus on education}

We will be introducing a number of features to the journal as part of this new focus on education in pediatric radiology.
Efforts will be directed at innovation and improvement of educational methods. A series of commentaries and reviews by education leaders will introduce topics, share experience and opinions, and review pertinent literature, opening new doors to how we think about education. We have reintroduced the "How-I-do-It" rubric for radiology educators to share their expertise and innovation. We hope to have a new focus with original research papers on education in radiology, and we have assembled a roster of reviewers with expertise in education to review such submissions and help the authors to optimize their contributions.

\section{Conclusion}

As leaders in education, we can have a profound impact on the field of radiology by attracting new talent, creating a new science in education, and transforming the way we teach and the way our students develop into master consultants in radiology. The journal's renewed focus on education aims to share the science of radiology education to do just that.

\section{Compliance with ethical standards}

Conflicts of interest None

Publisher's note Springer Nature remains neutral with regard to jurisdictional claims in published maps and institutional affiliations. 